\title{
Quantum tunneling of thermal protons through pristine graphene
}

Igor Poltavsky, Limin Zheng, Majid Mortazavi, and Alexandre Tkatchenko

Citation: The Journal of Chemical Physics 148, 204707 (2018); doi: 10.1063/1.5024317

View online: https://doi.org/10.1063/1.5024317

View Table of Contents: http://aip.scitation.org/toc/jcp/148/20

Published by the American Institute of Physics 


\title{
Quantum tunneling of thermal protons through pristine graphene
}

\author{
Igor Poltavsky, ${ }^{1}$ Limin Zheng, ${ }^{2}$ Majid Mortazavi, ${ }^{2}$ and Alexandre Tkatchenko ${ }^{1}$ \\ ${ }_{1}^{1}$ Physics and Materials Science Research Unit, University of Luxembourg, Luxembourg City L-1511, Luxembourg \\ ${ }^{2}$ Fritz-Haber-Institut der Max-Planck-Gesellschaft, Faradayweg 4-6, 14195 Berlin, Germany
}

(Received 31 January 2018; accepted 9 April 2018; published online 31 May 2018)

\begin{abstract}
Engineering of atomically thin membranes for hydrogen isotope separation is an actual challenge which has a broad range of applications. Recent experiments [M. Lozada-Hidalgo et al., Science 351, 68 (2016)] unambiguously demonstrate an order-of-magnitude difference in permeabilities of graphene-based membranes to protons and deuterons at ambient conditions, making such materials promising for novel separation technologies. Here we demonstrate that the permeability mechanism in such systems changes from quantum tunneling for protons to quasi-classical transport for heavier isotopes. Quantum nuclear effects exhibit large temperature and mass dependence, modifying the Arrhenius activation energy and Arrhenius prefactor for protons by more than $0.5 \mathrm{eV}$ and by seven orders of magnitude correspondingly. Our findings not only shed light on the separation process for hydrogen isotope ions passing through pristine graphene but also offer new insights for controlling ion transport mechanisms in nanostructured separation membranes by manipulating the shape of the barrier and transport process conditions. Published by AIP Publishing. https://doi.org/10.1063/1.5024317
\end{abstract}

\section{INTRODUCTION}

Atomically thin two-dimensional materials are increasingly being explored as a possible platform for developing novel separation technologies such as water desalination or proton-exchange membranes in fuel cells. ${ }^{1-16}$ Particular attention is given to the utilization of layered $2 \mathrm{D}$ materials for selective sieving of molecules, atoms, and ions. Recent experiments conducted by Geim's group have demonstrated that thermal protons (and deuterons) show appreciable conductance through a pristine graphene sheet (PGS) at ambient conditions. ${ }^{3,4}$ Remarkably, the experimentally deduced proton transport barrier of $0.78 \mathrm{eV}$ is at least $0.65 \mathrm{eV}$ lower than those predicted by electronic structure calculations ${ }^{17,18}$ for pristine graphene. Moreover, the difference in PGS areal conductivity for protons and deuterons cannot be explained by such calculations because the nuclear mass does not appear in the electronic Hamiltonian. ${ }^{19}$ In Ref. 20 it was shown that the hydrogenation of graphene, neglected in previous firstprinciples calculations, can lead to a substantial decrease in the barrier to less than $1 \mathrm{eV}$, which partially explains the experimental observations. However, the explanation of the substantial isotope effect was restricted up to now to the consideration of zero-point energy (ZPE) contributions to the height of the barrier ${ }^{20}$ and the energy of the initial state, ${ }^{4}$ while the nuclear quantum effects (NQE) beyond ZPE have been neglected.

In general, nuclear quantum effects strongly depend on the anharmonicity of realistic interatomic potentials and can qualitatively change the transport mechanism leading to various striking effects. Examples are a substantial deviation of the temperature dependence of the transmission rate from the classical Arrhenius-like behavior or even temperature-independent membrane permeability. ${ }^{21}$ In contrast to ZPE correction, where only the potential-energy profile near the classical transition state is relevant, the NQE beyond ZPE are dependent upon the entire shape of the potentialenergy surface (PES). Hence, to describe ionic transport, one must consider the ion-graphene interaction on ab initio level of accuracy as well as account for the thermal and quantum fluctuations of all atoms in the system. Existing methods for computing reaction rates ${ }^{22}$ are not yet practically applicable for such complex multidimensional quantum-mechanical problems. The development of new complementary approaches is required to unambiguously reveal the mechanism of proton/deuteron transport through PGS as well as other complex transport phenomena.

In this work, we develop a hierarchical quantummechanical methodology for directly computing membrane permeabilities. Using this framework, we demonstrate the qualitative difference in the transport mechanisms for protons and heavier hydrogen isotopes. First, we consider an optimal one-dimensional transport pathway when ions follow a linear trajectory perpendicular to the PGS through the center of a carbon ring [see Fig. 1(a)]. This pathway corresponds to the minimal potential energy barrier and yields the largest contribution to graphene permeability. We find that protons tunnel through PGS, while heavier isotopes follow quasi-classical transport pathways. As a result, the average energy of protons contributing to the transmission process is considerably lower than the height of the potential energy barrier. This leads to a large reduction (more than $0.5 \mathrm{eV}$ ) of the Arrhenius activation energy for protons ${ }^{3}$ as compared to the results of electronic structure calculations with classical nuclei. The difference in transport mechanisms is also responsible for the large proton-deuteron separation ratio. ${ }^{4}$ 


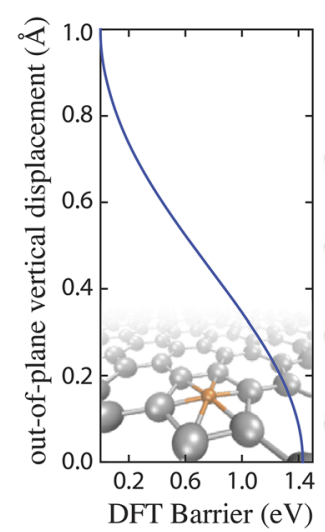

(a) $1 \mathrm{D}$ transition model (WKB)

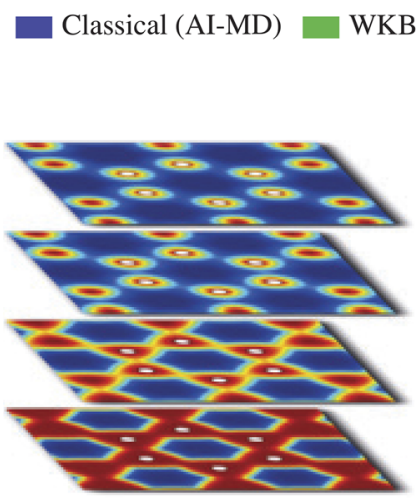

(b) Free energy thermodynamic integration (FETI)

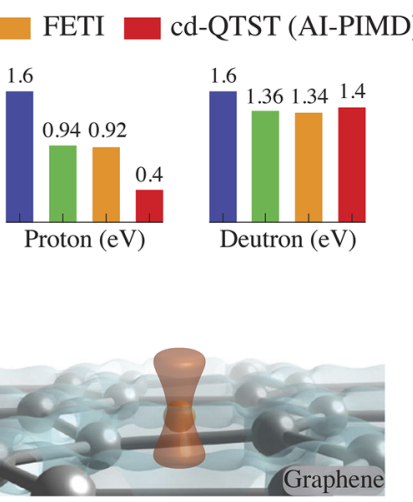

(c) Centroid-density quantum transition state theory (cd-QTST)

FIG. 1. Proton and deuteron transport through pristine graphene. Color bars show the value of the Arrhenius activation energy obtained with different approaches that are schematically explained in sub-figures (a)-(c). (a) The one-dimensional potential-energy barrier used for the transport model based on the WentzelKramers-Brillouin (WKB) approximation. (b) Examples of three-dimensional PES at different ion-graphene distances $(0.0,0.2,0.4,0.6 \AA)$ used for the free energy thermodynamic integration (FETI) approach. (c) System geometry used in AI-PIMD simulations within centroid-density quantum transition state theory (cd-QTST) method.

In the second step, we account for the transport pathways beyond a single linear trajectory by performing free energy thermodynamic integration (FETI) using full densityfunctional theory (DFT) Born-Oppenheimer (BO) potentialenergy surface (PES) with fixed membrane geometry [see Fig. 1(b)]. We have found that the presence of chemisorption sites, which can bind ions, leads to negligibly small average transmission coefficients, making bare PGS impenetrable for hydrogen ions. ${ }^{16}$ At the same time, blocking the chemisorption of $\mathrm{H}$ ions, for instance, by assuming pre-hydrogenation of graphene, leads to more than $10^{20}$ increase in transmission coefficients. Thus, the pre-hydrogenation of graphene or covering it with some material (such as $\mathrm{Nafion}^{3,4}$ ) plays a vital role in the transport process of $\mathrm{H}$ ions, defining not only the energy of an initial transition state ${ }^{3}$ but also affecting the permeability of graphene ${ }^{20}$-an effect which is attributed to specific experimental conditions.

In the final step, our full-dimensional ab initio imaginarytime Feynman-Kac path integral molecular dynamics (AI-PIMD) simulations ${ }^{23-26}$ demonstrate that the motion of carbon atoms in graphene is important for the transport process, concomitantly decreasing the proton activation energy [Fig. 1(c)].

All in all, our hierarchical quantum model (see Fig. 2) provides novel interpretations for recent key experimental observations, ${ }^{3,4}$ including (i) the quantum tunneling mechanism of proton transport, (ii) strong temperature dependence of the prefactor in the Arrhenius equation, (iii) the impact of chemisorption sites and covering material, and (iv) the role of mobile carbon atoms in hydrogen ion transport through PGS.

\section{ARRHENIUS EQUATION AND QUANTUM PROCESSES}

Transport experiments are often interpreted with the aid of the Arrhenius equation, which connects the rate constant $(k)$ to the inverse absolute temperature $(\beta)$,

$$
k=A \exp (-\beta \Lambda)
$$

Here $A$ is the pre-exponential factor and $\Lambda$ is the so-called Arrhenius activation energy - the minimal energy required for reactants to transform into products.

However, this equation is valid only for classical processes. Whenever quantum effects play a considerable role, a single energy cannot describe the corresponding reaction. In the case of transport, trajectories with different energies will contribute to the observed rates. Moreover, these contributions strongly depend upon temperature. This will result in a curvature in the Arrhenius plots and non-trivial temperature dependence of both $A$ and $\Lambda .{ }^{21}$

Below we will show that the thermal transport of protons through PGS at ambient conditions is strongly affected by tunneling, whereas heavier hydrogen ion isotopes follow nearly classical trajectories. Therefore, the pre-exponential factor $A$

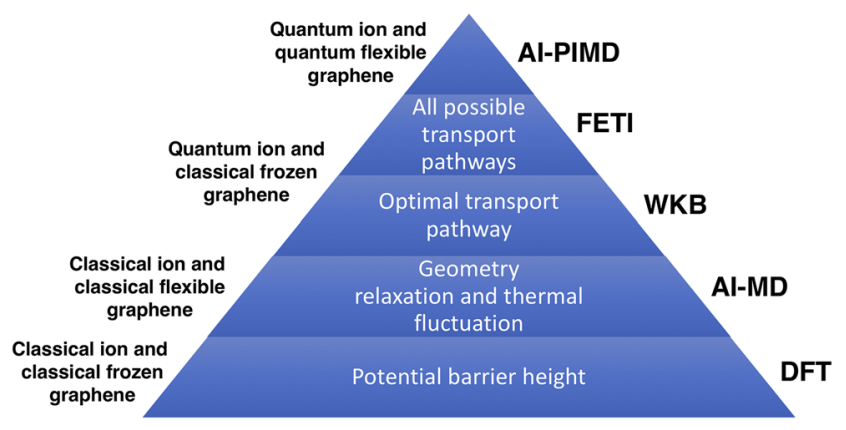

FIG. 2. The hierarchy of methods employed in this work. Density functional theory (DFT) and classical $a b$ initio molecular dynamics (AI-MD) treat nuclei classically and cannot explain the experimental observations. The Wentzel-Kramers-Brillouin (WKB) model and free energy thermodynamic integration (FETI) include NQE and describe the isotope effect but still overestimate the activation energy. Ab initio imaginary-time path integrals molecular dynamic (AI-PIMD) simulations allow us to explain both the activation energy reduction and isotope effect by consistent inclusion of NQE, PGS geometry relaxation, and thermal fluctuations of carbon atoms. 
and the activation energy $\Lambda$ in the Arrhenius equation (1) are temperature dependent for protons and different from those for deuterons or tritons. The value of $\Lambda$ obtained by using Eq. (1) for protons is not the minimal energy required for the transport process to occur. To emphasize this fact, we will use hereafter the term "Arrhenius activation energy" instead of activation energy.

The following terminology is used below in this article: (i) Arrhenius activation energy - the slope of a logarithm of a transport rate versus the inverse temperature. Its value coincides with a commonly used activation energy only in the case of classical processes. (ii) Quasi-classical transport process - the mean energy of the particles participating in such a transport process is close to the height of the barrier. (iii) Quantum transport process-the energy distribution of the particles participating in such a transport process is considerably affected by tunneling and substantially extends into the classically forbidden low-energy region.

\section{TRANSPORT ALONG AN OPTIMAL PATHWAY}

The possible permeability of PGS is an open controversial question. In some experiments ${ }^{16}$ protons are found to transfer only through rare, naturally occurring atomic defects. At the same time, experiments conducted by Geim's group ${ }^{3,4}$ clearly demonstrate appreciable thermal proton transport through a PGS at ambient conditions. An essential common part of both sets of experiments is the graphene layer. Thus, to reveal the potential permeability of pristine graphene-based membranes, the conditions when both experimentally observed situations may happen, and the transmission mechanisms, we focus on the thermal flux of hydrogen isotope ions through PGS in vacuum. We compute the direct observable-membrane permeability - which allows us to extract Arrhenius activation energies without invoking any additional assumptions about the value of pre-exponential coefficients. $3,4,27$

As a first step, we study the presumably most favorable transport pathway for a hydrogen isotope ion that follows a linear trajectory perpendicular to the PGS through the center of a carbon ring [see Fig. 1(a)]. This scenario corresponds to the lowest possible potential energy barrier $U(z)$ and yields an upper bound to the permeability of PGS with fixed geometry. Henceforth in this work, we obtain $U(z)$ from DFT calculations by employing the non-empirical exchangecorrelation functional of Perdew-Burke-Ernzerhof ${ }^{28}$ (PBE) and the Tkatchenko-Scheffler method ${ }^{29}$ to account for van der Waals interactions as implemented in the FHI-aims code. ${ }^{30}$ An optimized free-standing graphene geometry is used for all ion-graphene distances. The unit cell consists of 32 carbon atoms, and periodic boundary conditions are applied. Further details of the calculations are presented in Sec. $\mathrm{S} 1$ of the supplementary material. Note that the particular choice of the PBE functional does not lead to any specific functional dependent conclusions and allows us to employ both analytical and numerical methods. For instance, the employment of the computationally more expensive PBE0 functional leads to the increase of the height of the barrier by $0.1 \mathrm{eV}$ compared to PBE and modifies the isotope separation ratio by only $10 \%$. The obtained barrier height is also in good agreement with the barrier computed with optB88-vdW potential in Ref. 20 for the transition from the physisorbed state.

The transport process in this approximation is simplified to an effective one-dimensional (1D) problem of a transport along a reaction coordinate, which is defined by the distance between the ion and the graphene plane. An average transmission probability $k$ can be found as a ratio of passed and incident ion fluxes.

The flux of particles through the barrier can be found by employing a 1D transition-state model proposed in Ref. 21,

$$
j=\int_{0}^{\infty} v(p) f(p) T(p) d p
$$

where $v(p)=p / m, m$ is the particle mass, $p$ is its momentum, $T(p)$ is the transmission coefficient of the barrier, and $f(p)$ is the momentum probability distribution of the incoming flux of particles. Here we consider a thermal flux of particles, whose momentum distribution obeys the Maxwell-Boltzmann statistics (ions move freely from an infinite distance to the graphene plane interacting only with the potential barrier of the membrane),

$$
f(p)=\sqrt{\frac{\beta}{2 \pi m}} \exp \left(-\frac{\beta p^{2}}{2 m}\right) .
$$

The influence of the momentum distribution of ions in the incident flux on the transparency of the barrier and the isotope separation ratio will be discussed below.

The transmission coefficient $T$ of a single 1D barrier when the energy $E$ of a particle is smaller than the maximum height of the barrier $U_{\max }$ can be obtained using the Wentzel-KramersBrillouin (WKB) approximation, ${ }^{31}$

$$
T(E)=\exp \left\{-\frac{2}{\hbar} \sqrt{2 m} \int_{z_{1}(E)}^{z_{2}(E)} \sqrt{U(z)-E} d z\right\}
$$

Here $z_{i}(E)$ are the distances where $U\left(z_{i}\right)=E$. Since we are interested in temperatures which are much smaller than $U_{\max }$, we can neglect the over-barrier reflection, setting $T(E)=1$ for $E>U_{\max }$. Combining Eqs. (2)-(4), we obtain

$$
j_{\text {pass }}^{\mathrm{q}}(\beta)=j_{\text {pass }}^{\mathrm{c}}(\beta)+\sqrt{\frac{\beta}{2 \pi m}} \int_{\infty}^{0} T(U(z)) e^{-\beta U(z)} \frac{\partial U}{\partial z} d z,
$$

where $j_{\text {pass }}^{\mathrm{q}}$ and $j_{\text {pass }}^{\mathrm{c}}$ are the fluxes of particles passing the barrier computed within classical and quantum-mechanical approaches, respectively,

$$
j_{\text {pass }}^{\mathrm{c}}(\beta)=\frac{e^{-\beta U_{\max }}}{\sqrt{2 \pi m \beta}} .
$$

Finally, for the average transmission probability $k$, we have

$$
k^{\alpha}=\sqrt{2 \pi m \beta} j_{\text {pass }}^{\alpha}, \quad \alpha=\mathrm{c}, \mathrm{q} .
$$

Figure 3 shows the Arrhenius plot for protons, deuterons, and tritons in the temperature range $200-800 \mathrm{~K}$. One can see a pronounced deviation of the inverse temperature dependence of the transmission coefficient for a proton from the classical linear behavior for temperatures below $350 \mathrm{~K}$. With a 


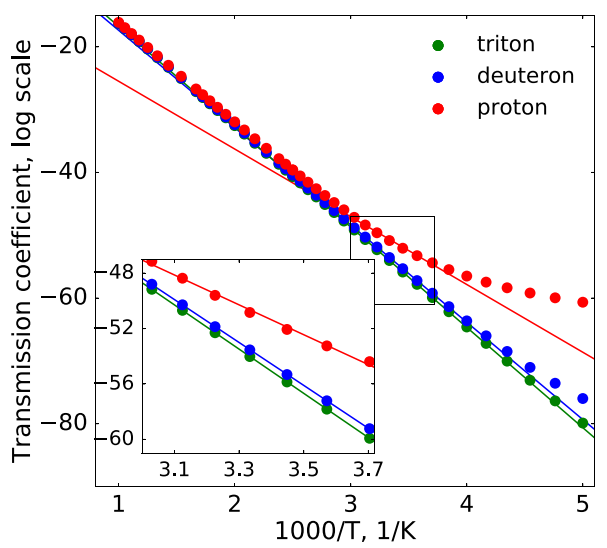

FIG. 3. Transmission coefficient as a function of inverse temperature for proton, deuteron, and triton transport through a pristine graphene layer for the temperature range $200-800 \mathrm{~K}$. Symbols are the results of the one-dimensional tunneling model (see the text), while solid lines of the same color are the best linear fitting in the experimentally relevant temperature range $270-330 \mathrm{~K}$ (see the inset).

further decrease in temperature, $k$ becomes weakly temperature dependent indicating the increasing role of tunneling transport mechanism, which is characterized by a complete independence of the transmission coefficient upon temperature. A similar nonclassical behavior for deuterons is observed for considerably smaller temperatures (below $250 \mathrm{~K}$ ) due to the larger mass of this isotope. In the experimentally relevant temperature range $270-330 \mathrm{~K}^{3}$ all three curves in the inset of Fig. 3 exhibit nearly linear behavior. For deuterons and tritons, this is caused by a quasi-classical nature of the transport process. In contrast, for protons, the linear shape of the Arrhenius plot is a result of a large compensation of the temperature dependent $\Lambda$ and $A$ in Eq. (1) and a small width of the temperature window employed in the experiment. At higher and lower temperatures, the linear extrapolation obtained from the temperature range 270-330 K (red solid line in Fig. 3) considerably deviates from the actual transmission coefficient (red symbols in Fig. 3) for protons.

The analytical expression for the transmission coefficient (see Sec. S1 of the supplementary material) allows us to compute separately the Arrhenius activation energy and the prefactor as a function of temperature literally repeating the experimental procedure. ${ }^{3}$ The results for $270-330 \mathrm{~K}$ are shown in Fig. 4 (wider temperature range of 200-800 K is presented in Sec. S2 of the supplementary material). One can see that within the considered temperature range the prefactor, $A$, for protons changes by seven orders of magnitude, while the Arrhenius activation energy increases by more than $0.4 \mathrm{eV}$. In contrast, for heavier isotopes, both $A$ and $\Lambda$ are much less temperature dependent. This indicates a qualitative difference in the leading mechanisms of proton and deuteron/triton transport. From Fig. 4 it also follows that accounting for prefactors is crucial to obtain the correct value of the isotope effect. The difference in the Arrhenius activation energies for protons and deuterons at $300 \mathrm{~K}$ would lead to a separation ratio of $\exp \left\{-\beta\left(\Lambda_{\text {proton }}-\Lambda_{\text {deuteron }}\right)\right\} \sim 5 \times 10^{6}$. Quantum prefactors yield a separation ratio $\left(A_{\text {proton }} / A_{\text {deuteron }}\right)$ $\times \exp \left\{-\beta\left(\Lambda_{\text {proton }}-\Lambda_{\text {deuteron }}\right)\right\} \sim 16$ in excellent agreement with experiment. ${ }^{4}$

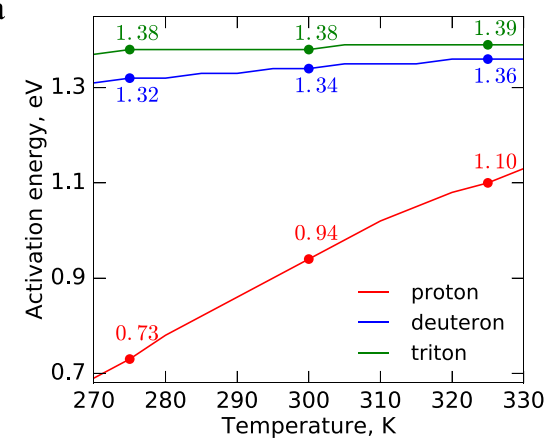

b

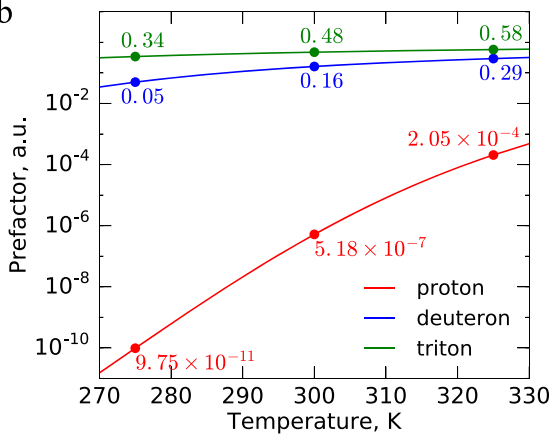

FIG. 4. Activation energies (a) and prefactors (b) extracted from the classical form of Arrhenius equation when it is applied for proton, deuteron, and triton tunneling through a pristine graphene layer along the optimal transport pathway.

To clarify the nature of transport mechanisms for different ions, we plot the contribution of ions to the transport process $[f(\epsilon) T(\epsilon)$ from Eq. (2)] as a function of their initial energy $\epsilon$. The results for thermal flow through PGS at room temperature are shown in Fig. 5. Clearly, the energy windows for permeation of protons and deuterons/tritons are quite different. The main contribution to the heavy hydrogen isotope transport process is due to ions whose energies are comparable to the height of the barrier $E_{\mathrm{DFT}}$. This hints on the quasi-classical nature of the transport process for deuterons and tritons. In this case, the reduction of the activation energy is mainly caused by ZPE effects. ${ }^{32}$ Indeed, the obtained value of the Arrhenius activation energy for deuterons of $1.36 \mathrm{eV}$ is in a good agreement with the results of ZPE calculations in Ref. 27. The value of the activation energy for tritons is found to be of $1.39 \mathrm{eV}$. The small difference in the

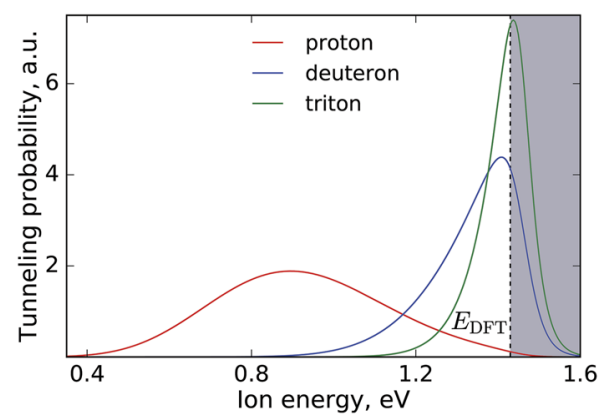

FIG. 5. Relative contribution to the tunneling process through PGS, in arbitrary units (a.u.), from ions with different initial energies $(\mathrm{eV})$ at $300 \mathrm{~K}$. 
activation energy shows that both isotopes obey a similar transport mechanism.

For protons, we observe a qualitatively different behavior. The energies of transmitting particles are considerably lower than the height of the potential energy barrier which corresponds to the so-called tunneling regime. The obtained value of Arrhenius activation energy of $0.94 \mathrm{eV}$ is caused by the quantum tunneling of protons through the graphene layer. This conclusion is also supported by the fact that the crossover temperature between shallow and deep tunneling regimes ${ }^{33}$ for protons within the considered one-dimensional potential profile is $320 \mathrm{~K}$. The fact that the crossover temperature falls within the range of the temperatures of practical interest makes the description of proton transport through PGS an especially challenging problem. All the existing methods for computing transmission rates demonstrate the largest errors within this regime. In Secs. IV and V, we will employ two more independent approaches to verify the conclusions obtained within our 1D model.

An important consequence of the quantum tunneling nature of proton transport is the critical influence of the shape of the potential energy barrier on the permeability of the system. The transmission coefficient can be varied by modifying the shape of the barrier without changing its height, ${ }^{34}$ that is, without introducing defects in graphene. This explains the increase in permeability observed in experiments ${ }^{3}$ upon decorating the PGS by nano-particles. A similar behavior should be obtained in the presence of electric fields. Evidently, the field which drives the ion flow through the graphene layer distorts the symmetry of the barrier suppressing the backscattering process.

\section{THREE-DIMENSIONAL TRANSPORT PROBLEM}

The 1D transport model described in Sec. III has provided insights into the ion transport mechanisms, but it does not account for several important factors. First and foremost, the degrees of freedom transverse to the reaction coordinate, which may play a nontrivial role, have been neglected until now. ${ }^{32}$ To address this issue, we compute the transport rate constant, $k$, by performing a free energy thermodynamic integration (FETI) using a three-dimensional (3D) Born-Oppenheimer DFT PES [for details of the calculations see Fig. 1(b) and Secs. S3 and S4 of the supplementary material],

$$
k=e^{-\beta \Delta F} .
$$

Here $\Delta F$ is the free energy change during the transport process,

$$
\Delta F=-\int_{\infty}^{0}\langle\vec{f}\rangle_{z} d \vec{z}
$$

where $\langle\vec{f}\rangle$ is the average force acting on the ion displaced from the graphene plane and $\vec{z}$ is the displacement vector pointing out of the graphene plane. The origin of the coordinates, $z=0$, is assumed to be inside PGS. The averaging $\langle\ldots\rangle_{z}$ has been done by fixing the $z$ coordinate of the centroid of the ion and performing imaginary-time path integral simulations using the precomputed PES. In contrast to the model described in Sec. III, the other two coordinates of the ion are not constrained, and the trajectories of ions can cross the carbon ring at any point. Similar to Sec. III, we determine $k$ as a transport probability rather than the transport frequency, which explains the absence of a prefactor in Eq. (8). Note that in this section the geometry of the graphene layer is also fixed and does not depend upon the position of the ion. The role of the carbon atoms' motion and PGS geometry optimization will be considered in Sec. VI.

By performing FETI we account for all possible transmission trajectories, also taking into consideration the chemisorption of ions on carbon atoms at a distance of $1.1 \AA$ from the graphene plane. The chemisorption sites demonstrate large binding energy of $1.7 \mathrm{eV}$ and have large cross-sectional area perpendicular to the graphene plane. The ions, trapped inside such sites, cannot participate in the transport process. As a result, PGS will behave as an impenetrable membrane for protons at the initial stage of the transport process. This is fully supported by the results of our simulations presented in Table I, where it can be seen that chemisorption leads to negligibly small average transmission coefficients.

However, effective blocking (saturation) of chemisorption sites in a given experiment may lead to qualitatively different observations. Such blocking can arise due to several factors, including the hydrogenation of graphene ${ }^{20}$ or the presence of covering materials (Nafion, etc.) inhibiting chemical bond formation. To mimic the blocking of chemisorption sites and to avoid unnecessary speculations about the particular blocking mechanisms and the nature of the initial transport state, we introduce the following approximation. We assume that for any ion-graphene distance $z$ [see Figs. 1(a) and 1(b)] the minimum of the interaction energy $U_{\min }(z)$ lies on a straight line passing through the center of a carbon ring. Hence, whenever at a given point $(x, y, z)$ the value of the interaction energy is lower than $U_{\min }(z)$, we set $U(x, y, z)=U_{\min }(z)$, otherwise we leave it unchanged. This allows us to retain the repulsion of an ion from carbon atoms at small ion-graphene distances $\leq 0.8 \AA$ and to avoid the attraction to chemisorption sites at larger $z$. As a result, we observe an increase of average transmission coefficients by more than 25 orders of magnitude (see Table I) and the values becoming comparable to the predictions of the 1D model. A small reduction of the transmission coefficient is caused by less favorable pathways than the linear trajectory passing through the centers of carbon hexagons. Remarkably, the isotope effect does not change qualitatively within the $3 \mathrm{D}$ model as compared to the $1 \mathrm{D}$ case and both calculations yield the same results for the Arrhenius activation energy (within $20 \mathrm{meV}$ ). Namely, FETI yields $\Lambda=0.92 \mathrm{eV}$ for protons and $1.34 \mathrm{eV}$ for deuterons.

TABLE I. Proton and deuteron reaction rate constants and their ratio at $300 \mathrm{~K}$. WKB denotes the 1D transport model described above, while FETI implies the free energy thermodynamic integration approach with and without blocking the chemisorption sites.

\begin{tabular}{lccc}
\hline & FETI & FETI (blocked chem.) & WKB \\
\hline Proton & $3.1 \times 10^{-50}$ & $1.9 \times 10^{-24}$ & $7.1 \times 10^{-23}$ \\
Deuteron & $2.0 \times 10^{-52}$ & $7.8 \times 10^{-26}$ & $4.3 \times 10^{-24}$ \\
Ratio & 155 & 24.4 & 16.5 \\
\hline \hline
\end{tabular}




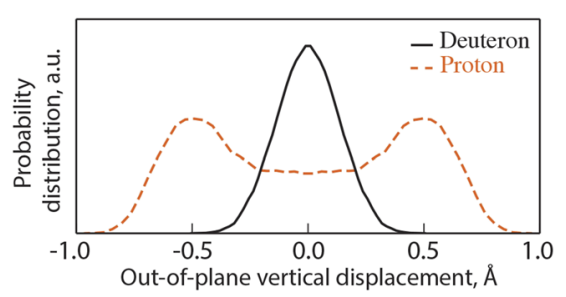

FIG. 6. Probability distributions in arbitrary units (a.u.) to find a proton/deuteron contributing to the transition state at a given distance from the graphene plane. The simulations have been preformed at the temperature of $300 \mathrm{~K}$.

To understand why the transmission coefficients obtained within WKB and FETI methods are in such a good agreement, we plot the probability of locating a proton and a deuteron contributing to the transition state as a function of the distance from the graphene surface (see Fig. 6). The inclusion of NQE leads to a substantial delocalization of protons with the probability maximum located around $0.5 \AA$ away from the graphene layer. This suggests that the in-plane ionic quantum fluctuations, which can considerably affect ionic transport in nanoporous materials, ${ }^{32}$ are of minor importance for proton tunneling through graphene. In contrast, the deuteron wavefunction is much less delocalized in the direction perpendicular to the graphene plane. Deuterons come closer to the graphene plane than protons during the transport process. The effective repulsion caused by in-plane ionic quantum fluctuations becomes essential. This leads to a more significant difference in the deuteron reaction rate constant predicted within the one-dimensional WKB model and the threedimensional FETI approach comparing to the case of proton transport.

Figure 6 also demonstrates that protons and deuterons, initially located at a distance larger than $\sim 0.8 \AA$ from the graphene plane, do not contribute appreciably to the transport process. This provides further evidence in favor of the conclusion that the hydrogen ions trapped at chemisorption sites at a distance of $\sim 1.1 \AA$ from the graphene layer cannot penetrate through a PGS.

\section{CALCULATING THE TRANSPORT BARRIER WITH MOLECULAR DYNAMICS}

To further asses the role of thermal and quantum fluctuations of the carbon atoms constituting the PGS in proton/deuteron transport process, we have performed classical and quantum $a b$ initio (AI) molecular dynamics (MD) simulations. The barrier $\Lambda$ is computed within the centroid-density quantum transition state theory (cd-QTST) as

$$
\Lambda=\left\langle E_{\text {reacting complexes }}\right\rangle-\left\langle E_{\text {reactants }}\right\rangle \text {. }
$$

Here $\left\langle E_{\text {reacting complexes }}\right\rangle$ is the average energy of a reacting complex and $\left\langle E_{\text {reactants }}\right\rangle$ is the average energy of reactants, i.e., free ion and free-standing PGS. We define the reacting complex by constraining the vertical positions of the hydrogen isotope and two carbon atoms in the surrounding graphene hexagon. Other degrees of freedom were allowed to fluctuate freely. Trajectory snapshots of AI-MD and AI-PIMD simulations for deuteron and proton in the transition state are shown in Fig. 7 (Multimedia view). These snapshots clearly demonstrate a qualitative difference in the delocalization of protons and deuterons in the transition state which leads to different transport mechanisms. Details of the simulations are presented in Sec. S5 of the supplementary material.

In full agreement with previous calculations, ${ }^{17,18}$ where only the electronic subsystem has been considered on a quantum-mechanical level, our AI-MD equilibrium proton transport barrier is $1.6 \mathrm{eV}$. The value of the classical AI-MD barrier is by $0.2 \mathrm{eV}$ larger than that predicted by DFT calculations. This well-known effect is a result of ionmembrane repulsion inside the graphene plane caused by atomic motions.

In our imaginary-time Feynman-Kac path integral molecular dynamics (PIMD) simulations, we define the transmission state by fixing the out-of-plane coordinate of centroids for the hydrogen ion and two carbon atoms in the surrounding graphene hexagon. All the other simulation details are similar to classical AI-MD simulations. To compute the average energies entering Eq. (10), we utilize a recently developed perturbed path integral (PPI) approach. ${ }^{35}$ This method is a combination of conventional $a b$ initio imaginary-time path integral molecular dynamics simulations with a posteriori corrections for thermodynamic observables using perturbation theory. The PPI method enables a convergence of $\sim 10 \mathrm{meV}$ for the total energy of the considered system with respect to the number of beads at ambient conditions using AI-PIMD trajectories with only 10 beads. Such convergence is an order of magnitude more accurate compared to the conventional second-order PIMD method and is required for a quantitative description of the tunneling process.

By allowing graphene atoms to move and fluctuate freely, we obtain the values of $\Lambda$ for protons and deuterons of 0.4 and $1.4 \mathrm{eV}$ correspondingly. Comparing the FETI activation energy for protons and the current result, one can see that strong interaction between protons and graphene at characteristic tunneling distances of $\sim 0.5 \AA$ makes the separation of graphene and ions into two subsystems rather inaccurate.

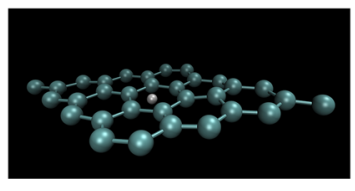

(a)

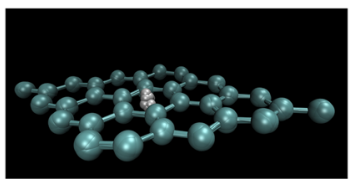

(b)

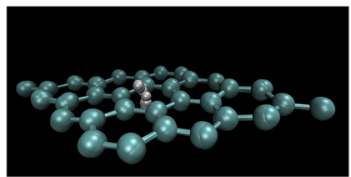

(c)

FIG. 7. Trajectory snapshots of (a) AI-MD and AI-PIMD simulations for (b) deuteron and (c) proton in the transition state. Multimedia views: https://doi.org/10.1063/1.5024317.1; https://doi.org/10.1063/1.5024317.2; https://doi.org/10.1063/1.5024317.3 
Importantly, the reduction of $\Lambda$ for protons is not a result of graphene geometry relaxation ignored in FETI calculations. The same relaxation is present in AI-PIMD simulations for deuterons where, due to quasi-classical nature of the transport process, the difference between $\Lambda$ obtained within these two methods is only $60 \mathrm{meV}$.

We remark that the value of $\Lambda$ for protons computed by employing Eq. (10) is not exactly the Arrhenius activation energy. Indeed, cd-QTST may be inaccurate due to strong quantum fluctuations of protons and the anisotropy of the barrier caused by the motion of carbon atoms. ${ }^{21}$ Unfortunately, the fact that the crossover temperature is within the temperature range of interest makes the thermal protons transport through PGS a challenging problem for any existing method for computing reaction rates. ${ }^{22}$ Another complication preventing direct ab initio calculations of reaction rates by employing more sophisticated methods than cd-QTST is the undefined mechanism of blocking of the chemisorption sites. Even the hydrogenation can happen in many different ways. ${ }^{20} \mathrm{Nev}-$ ertheless, more than the twofold reduction of the Arrhenius activation energy obtained within cd-QTST compared to quantum calculations with fixed carbon atoms or classical AI-MD simulations unambiguously indicates the strong mutual influence of ion and carbon quantum fluctuations. These fluctuations should considerably affect the transmission rates and can further decrease the activation energy.

\section{DISCUSSION}

Table II summarizes the results for the Arrhenius activation energy $\Lambda$ obtained within different methods employed in this paper.

Accounting for the quantum nature of an ion within WKB and FETI approaches leads to more than $0.5 \mathrm{eV}$ decrease of the Arrhenius activation energy. Note that the results of our one- and three-dimensional calculations are in very good mutual agreement when the chemisorption sites are assumed to be blocked. Importantly, the shape of the BO PES for ion-graphene distances smaller than $0.8 \AA$, which defines the transmission state (see Fig. 6), is determined by ion-graphene repulsion and cannot be substantially affected by a particular mechanism for blocking of the chemisorption sites. Hence, the obtained qualitative difference in proton and deuteron transport processes and the large decrease of the Arrhenius activation energy for protons is independent of particular experimental conditions (the presence of water and Nafion and hydrogenation of graphene). Obviously, in the experiment, the interaction of ions with the surrounding atoms and molecules leads to the change of the energy of the initial state. The ions can be held tightly by either Nafion or another water

TABLE II. Proton and deuteron Arrhenius activation energies (in eV) obtained within different approaches used in this article. The FETI results correspond to PES where the chemisorption sites are blocked. The DFT barrier values correspond to the transport from a physisorbed state.

\begin{tabular}{lcccccc}
\hline \hline & DFT & AI-MD & AI-PIMD & WKB & FETI & Exp. \\
\hline Proton & 1.43 & 1.6 & 0.4 & 0.94 & 0.92 & 0.78 \\
Deuteron & 1.43 & 1.6 & 1.4 & 1.36 & 1.34 & $\ldots$ \\
\hline \hline
\end{tabular}

molecule, which could potentially stabilize the initial state and, therefore, yield a higher overall barrier. On the other hand, the experimental observation of the proton transport at small bias fields suggests the presence of the mechanisms to overcome the binding of the ions to their carriers, while the nature of such mechanisms remains unclear. Hence, in principle, the energy of the initial state can be larger or smaller compared to the energy of a free proton. This may explain the overestimation of the activation energy obtained within our FETI method $(0.92 \mathrm{eV})$, where the initial state is considered to be a vacuum, as compared to the experimental value of $0.78 \mathrm{eV}$. Another reason may be the change of the height of the barrier, for instance, caused by the hydrogenation of the graphene surface.

From Fig. 5, it follows that one can modify the proton/deuteron separation ratio by changing the energy distribution of ions in the incident flux. For instance, the protons with energies below $\sim 0.4 \mathrm{eV}$ are reflected by the membrane. Thus, different behavior of the proton transport may be observed for bias voltages below and above $\sim 0.4 \mathrm{eV}$. For instance, for voltage biases above $\geq 0.4 \mathrm{eV}$ or due to the acquiring of an additional energy by the ion during the dissociation of an initial state, possible proton transport pathways with energies below the bias threshold will be excluded from the transmission process. This will reduce the proton-deuteron separation ratio. In contrast, cutting out the ions with energies above $\sim 1 \mathrm{eV}$ will make the membrane impenetrable for deuterons without noticeable changes in the transmission rate for protons. This can be used to increase the proton/deuteron separation ratio to, theoretically, any desired value. In practice, the efficiency of such a mechanism will be a limiting factor.

\section{SUMMARY}

Our first-principles quantum-mechanical approach has revealed the pronounced quantum nature of thermal proton transport through pristine graphene. We predict a substantial difference of $0.5 \mathrm{eV}$ in the Arrhenius activation energies for protons and deuterons and rationalize the experimentally measured isotope effect, which is strongly influenced by the mass-dependent prefactor in the Arrhenius equation. We found that in order to observe non-negligible ion transport, chemisorption on the graphene surface has to be blocked. The motion of carbon atoms in graphene is also an important factor, yielding considerable reduction in the Arrhenius activation energy for protons, compared to a fixed membrane approximation. Further theoretical developments are needed to obtain accurate ionic transmission coefficients from full-dimensional $a b$ initio calculations with an explicit mechanism of blocking chemisorption, an account for thermal and quantum fluctuations of membrane atoms, the presence of electric fields in the system, and ion-ion interaction. The incorporation of experimental conditions in simulations should reveal more subtle but potentially very important features of the transport process and control mechanisms for practical applications of hydrogen isotope ion tunneling.

\section{SUPPLEMENTARY MATERIAL}

See supplementary material for all the technical details of calculations employed in this work. Video files S1.mp4, 
S2.mp4, and S3.mp4 visualize AI-MD and AI-PIMD trajectories for deuterons and protons in the transition state (ion centroid is fixed in the plane of the graphene sheet), respectively. The PES.zip archive contains the PES code employed in Sec. IV.

\section{ACKNOWLEDGMENTS}

I.P. and A.T. acknowledge financial support from the Luxembourg National Research within the FNR-CORE program (Grant No. FNR-11360857).

${ }^{1}$ J. M. H. Kroes, A. Fasolino, and M. I. Katsnelson, Phys. Chem. Chem. Phys. 19, 5813 (2017).

${ }^{2}$ L. Tsetseris and S. Pantelides, Carbon 67, 58 (2014).

${ }^{3}$ S. Hu, M. Lozada-Hidalgo, F. Wang, A. Mishchenko, F. Schedin, R. Nair,

E. Hill, D. Boukhvalov, M. Katsnelson, R. Dryfe, I. Grigorieva, H. Wu, and A. Geim, Nature 516, 227 (2014).

${ }^{4}$ M. Lozada-Hidalgo, S. Hu, O. Marshall, A. Mishchenko, A. Grigorenko, R. Dryfe, B. Radha, I. Grigorieva, and A. Geim, Science 351, 68 (2016).

${ }^{5}$ R. Joshi, P. Carbone, F. Wang, V. Kravets, Y. Su, I. Grigorieva, H. Wu, A. Geim, and R. Nair, Science 343, 752 (2014).

${ }^{6}$ B. Mi, Science 343, 740 (2014).

${ }^{7}$ J. S. Bunch, S. S. Verbridge, J. S. Alden, A. M. van der Zande, J. M. Parpia, H. G. Craighead, and P. L. McEuen, Nano Lett. 8, 2458 (2008).

${ }^{8}$ K. Celebi, J. Buchheim, R. M. Wyss, A. Droudian, P. Gasser, I. Shorubalko, J.-I. Kye, C. Lee, and H. G. Park, Science 344, 289 (2014).

${ }^{9}$ S. P. Koenig, L. Wang, J. Pellegrino, and J. S. Bunch, Nat. Nanotechnol. 7, 728 (2012).

${ }^{10}$ S. C. O'Hern, C. A. Stewart, M. S. Boutilier, J.-C. Idrobo, S. Bhaviripudi, S. K. Das, J. Kong, T. Laoui, M. Atieh, and R. Karnik, ACS Nano 6, 10130 (2012).

${ }^{11}$ H. W. Kim, H. W. Yoon, S.-M. Yoon, B. M. Yoo, B. K. Ahn, Y. H. Cho, H. J. Shin, H. Yang, U. Paik, and S. Kwon, Science 342, 91 (2013).

${ }^{12} \mathrm{H}$. Li, Z. Song, X. Zhang, Y. Huang, S. Li, Y. Mao, H. J. Ploehn, Y. Bao, and M. Yu, Science 342, 95 (2013).
${ }^{13}$ S. Garaj, W. Hubbard, A. Reina, J. Kong, D. Branton, and J. Golovchenko, Nature 467, 190 (2010).

${ }^{14}$ R. Devanathan, Nat. Nanotechnol. 12, 500 (2017).

${ }^{15}$ D. Cohen-Tanugi and J. C. Grossman, Nano Lett. 12, 3602 (2012).

${ }^{16}$ J. L. Achtyl et al., Nat. Commun. 6, 6539 (2015).

${ }^{17}$ W. L. Wang and E. Kaxiras, New J. Phys. 12, 125012 (2010).

${ }^{18}$ M. Miao, M. B. Nardelli, Q. Wang, and Y. Liu, Phys. Chem. Chem. Phys. 15, 16132 (2013).

${ }^{19}$ R. G. Parr and W. Yang, Density-Functional Theory of Atoms and Molecules (Oxford University Press, New York, 1989).

${ }^{20}$ Y. Feng, J. Chen, W. Fang, E.-G. Wang, A. Michaelides, and X.-Z. Li, J. Phys. Chem. Lett. 8, 6009 (2017).

${ }^{21}$ P. D. Pacey, J. Chem. Phys. 71, 2966 (1979).

${ }^{22}$ J. O. Richardson and S. C. Althorpe, J. Chem. Phys. 131, 214106 (2009).

${ }^{23}$ R. P. Feynman and A. R. Hibbs, Quantum Mechanics and Path Integrals (McGraw-Hill, New York, 1965).

${ }^{24}$ B. J. Berner and D. Thirumalai, Annu. Rev. Phys. Chem. 37, 401 (1986).

${ }^{25}$ M. E. Tuckerman, D. Marx, M. L. Klein, and M. Parrinello, J. Chem. Phys. 104, 5579 (1996).

${ }^{26}$ D. Marx and M. Parrinello, J. Chem. Phys. 104, 4077 (1996).

${ }^{27}$ Q. Zhang, M. Ju, L. Chen, and X. C. Zeng, J. Phys. Chem. Lett. 7, 3395 (2016).

${ }^{28}$ J. P. Perdew, K. Burke, and M. Ernzerhof, Phys. Rev. Lett. 77, 3865 (1996).

${ }^{29}$ A. Tkatchenko and M. Scheffler, Phys. Rev. Lett. 102, 073005 (2009).

${ }^{30}$ V. Blum, R. Gehrke, F. Hanke, P. Havu, V. Havu, X. Ren, K. Reuter, and M. Scheffler, Comput. Phys. Commun. 180, 2175 (2009).

${ }^{31}$ B. C. Hall, Quantum Theory for Mathematicians (Springer, New York, 2013).

${ }^{32}$ M. I. Hernández, M. Bartolomei, and J. Campos-Martínez, J. Phys. Chem. A 119, 10743 (2015).

${ }^{33}$ J. O. Richardson, "Ring-polymer approaches to instanton theory," Ph.D. thesis, University of Cambridge, 2012.

${ }^{34}$ L. D. Landau and E. M. Lifshitz, Quantum Mechanics: Non-Relativistic Theory, 3rd ed. (Butterworth-Heinemann, Oxford, 1981).

${ }^{35}$ I. Poltavsky and A. Tkatchenko, Chem. Sci. 7, 1368 (2016). 